\title{
Chromosome-Scale Genome Assembly of Fusarium oxysporum Strain Fo47, a Fungal Endophyte and Biocontrol Agent
}

\author{
Bo Wang, ${ }^{1}$ Houlin Yu, ${ }^{2,3}$ Yanyan Jia, ${ }^{1}$ Quanbin Dong, ${ }^{4}$ Christian Steinberg, ${ }^{5}$ Claude Alabouvette, ${ }^{5}$ \\ Veronique Edel-Hermann, ${ }^{5}$ H. Corby Kistler, ${ }^{6}$ Kai Ye, ${ }^{1,4, \dagger}$ Li-Jun Ma, ${ }^{2,3, \dagger}$ and Li Guo ${ }^{1,4, \dagger}$ \\ ${ }^{1}$ MOE Key Laboratory for Intelligent Networks \& Network Security, Faculty of Electronic and Information \\ Engineering, Xi'an Jiaotong University, Xi'an 710049, China \\ ${ }^{2}$ Graduate Program of Plant Biology, University of Massachusetts Amherst, Amherst, MA 01003, U.S.A. \\ ${ }^{3}$ Department of Biochemistry and Molecular Biology, University of Massachusetts Amherst, Amherst, MA \\ 01003, U.S.A. \\ ${ }^{4}$ School of Life Science and Technology, Xi'an Jiaotong University, Xi'an 710049 China \\ ${ }^{5}$ Agroécologie, AgroSup Dijon, INRA, University of Bourgogne Franche-Comté, F-21000 Dijon, France \\ ${ }^{6}$ USDA ARS Cereal Disease Laboratory, University of Minnesota, St. Paul, MN 55108, U.S.A.
}

\section{Abstract}

Here, we report a chromosome-level genome assembly of Fusarium oxysporum Fo47 (12 pseudomolecules; contig $\mathrm{N}_{50}$ : $4.52 \mathrm{Mb}$ ), generated using a combination of PacBio long-read, Illumina paired end, and high-throughput chromosome conformation capture sequencing data. Although F. oxysporum causes vascular wilt to over 100 plant species, the strain Fo47 is classified as an endophyte and is widely used as a biocontrol agent for plant disease control. The Fo47 genome carries a single accessory chromosome of $4.23 \mathrm{Mb}$, compared with the reference genome of $F$. oxysporum f. sp. lycopersici Fol4287. The high-quality assembly and annotation of the Fo47 genome will be a valuable resource for studying the mechanisms underlying the endophytic interactions between $F$. oxysporum and plants as well as for deciphering the genome evolution of the F. oxysporum species complex.

\section{Genome Announcement}

The filamentous ascomycete fungus Fusarium oxysporum represents a species complex of diverse members that cause soil-borne vascular wilt diseases to over 100 plant species in a host-specific fashion (Edel-Hermann and Lecomte 2019; Kistler 1997; Ma et al. 2013). Strains pathogenic to specific host species are classified as formae speciales (f. $\mathrm{sp}$.), and, together with related nonpathogenic strains, they are collectively known as the $F$. oxysporum species complex (Edel-Hermann and Lecomte 2019; Kistler 1997; Michielse and Rep 2009). Although notorious as plant pathogens, F. oxysporum strains are commonly plant endophytes often beneficial to host plants (Alabouvette et al. 2009; de Lamo and Takken 2020). Fo47, a strain isolated from plant disease-suppressive soils (Alabouvette 1986), grows endophytically and is widely used as a biological control agent for preventing vascular wilt of tomato, caused by F. oxysporum f. sp. lycopersici (Alabouvette et al. 2009), and other plant diseases as well (He et al. 2002; Paparu et al. 2007; Veloso and Díaz 2012). In general, beneficial microbes

${ }^{\dagger}$ Corresponding authors: K. Ye; kaiye@ @jtu.edu.cn, L.-J. Ma; lijun@ biochem.umass.edu, and

L. Guo; guo_li@xjtu.edu.cn

The author(s) declare no conflict of interest.

Accepted for publication 16 June 2020.

(C) 2020 The American Phytopathological Society

\section{Funding}

This project was supported by the National Natural Science Foundation of China (31701739 and 31970317), Fundamental Research Fund of Xi'an Jiaotong University, and National Key R\&D Program of China (2018YFC0910400).

\section{Keywords}

biocontrol, fungal endophyte, Fusarium oxysporum, genome assembly, plant-fungal interactions 
contribute to the fitness of host plants, potentially through diverse mechanisms including induced systemic resistance, promoting nutrient assimilation, and conferring antagonisms against microbial pathogens (Alabouvette et al. 2009; Aimé et al. 2013; de Lamo and Takken 2020; Pieterse et al. 2014; Van der Ent et al. 2009). The biocontrol effect of Fo47 is widely exploited in agriculture, but the molecular mechanisms behind it remain poorly understood. Decoding the genome of Fo47 will provide a valuable resource, not only facilitating dissection of such mechanisms but also contributing to comparative genomic studies targeted toward understanding the genome evolution of the $F$. oxysporum species complex in terms of endophytism, pathogenesis, and host specificity. A previous genome assembly was deposited at the National Center for Biotechnology Information (NCBI) under accession number GCA_000271705.2.

High-molecular weight genomic DNA was extracted from 4-day-old Fo47 mycelia using the CTAB (cetyltrimethylammonium bromide) method. A total of $6.85 \mathrm{~Gb}$ of paired-end short sequence reads $(2 \times 150 \mathrm{bp})$ and $18.09 \mathrm{~Gb}$ of single-molecule long-read sequence reads were generated, using the Illumina NovaSeq 6000 and PacBio SMRT RS II platforms, respectively. K-mer (17 mer) frequency analysis of $6.85 \mathrm{~Gb}$ of Illumina reads (138x coverage) using the Jellyfish-GenomeScope pipeline with default parameters (Marçais and Kingsford 2011; Vurture et al. 2017) revealed an estimated genome size of $49.54 \mathrm{Mb}$ for Fo47. The 18.09-Gb (341× coverage) PacBio SMRT data (1.24 million reads) has a read length $\mathrm{N}_{50}$ of $18,156 \mathrm{bp}$ after filtering. We then assembled a Fo47 genome from the PacBio reads using the assembler NextDenovo v2.0, with parameters 'read_cutoff = 1k' and 'seed_cutoff $=34,834$ '. The PacBio assembly was further polished by Illumina PE reads using Pilon v. 1.22 with parameters '-changes-vcf-frags' (Walker et al. 2014) to correct 2,040 (0.004\%) erroneous positions. To make the chromosome-scale assembly, the contigs were scaffolded using chromatin contact maps produced by high-throughput chromosome conformation capture (Hi-C) reads. Basically, a single Hi-C library prepared from cross-linked chromatins of fungal cells, using a standard Hi-C protocol (Belton and Dekker 2015), were sequenced by NovaSeq 6000 to yield $5.76 \mathrm{~Gb}(115 \times$ coverage $)$ Illumina $\mathrm{PE}$ reads. The $\mathrm{Hi}-\mathrm{C}$ data were used to anchor all contigs on 12 pseudomolecules (chromosomes), using Juicer v. 1.5 with default parameters (Durand et al. 2016b) and Juicebox v. 1.11 .08 (Durand et al. 2016a), followed by manual checks. The number of chromosomes was determined by centromeric interaction regions detected in $\mathrm{Hi}-\mathrm{C}$ contacted map figures (Marbouty et al. 2014).

The final assembly of the strain Fo47 genome is $50.36 \mathrm{Mb}$ placed in 12 chromosomes, proximate to its estimated genome size. Each chromosome is represented in a single contig, with a contig $\mathrm{N}_{50}$ of $4.52 \mathrm{Mb}$, demonstrating the high continuity of this assembly (Table 1). Of 12 chromosomes, nine had more than three copies of the telomeric repeat unit TTAGGG on both ends, whereas the other three contained those copies on only one end, indicating a highquality Fo47 genome assembly of chromosome level. Compared with a previous Fo47 genome assembly (GenBank accession GCA_000271705.2) at NCBI (Table 1), the most important improvement concerns chromosome 7 , as 73 contigs (3.96 $\mathrm{Mb})$ of the initial assembly can be aligned to the single 4.23-Mb Chr7 (telomeric repeats on both ends) of the new assembly, using Mummer v. 3.1 with default parameters (Kurtz et al. 2004).

For genome annotation, GeneMark-ES with parameters '-ES-fungus' (Borodovsky and Lomsadze 2011) was first used for de novo gene-structure prediction. A Fusarium-specific gene model was then used to train Augustus v. 3.1 (Stanke and Morgenstern 2005). MAKER2 pipeline (Cantarel et al. 2008) was used to find protein-coding genes integrating gene models predicted from GeneMark-ES and Augustus and protein sequences of the F. oxysporum reference genome of a tomato-pathogenic isolate, Fol4287 (GenBank assembly accession GCA_000149955.2) (Ma et al. 2010), with the RepeatMasker v. 4.0.7 (Tarailo-Graovac and Chen 2009) option on to find and mask repetitive elements. In total, the Fo47 genome has 17,127 protein-coding genes and $3.42 \%$ repeat content (Table 1 ). The genome assembly completeness was assessed by BUSCO (benchmarking universal single copy ortholog) v. 1.22 with parameters '-m OGS' (Simão et al. 2015), using proteome sequences showing BUSCO score of $99 \%$ based on the 'fungi_odb9' library (Table 1), suggesting a high degree of completeness for this Fo47 genome assembly.

Compared with the reference genome Fol4287 (Ma et al. 2010), Fo47 has a smaller genome size and fewer genes and repeat elements (Table 1). A whole-genome sequence alignment between Fo47 and Fol4287 using Mummer v. 3.1 with default parameters (Kurtz et al. 2004) reveals that the size of sequence mapped to the core genome of Fol4287 (11

Vol. 33, No. 9, $2020 / 1109$ 
Table 1. Summary of the genome assembly and annotation statistics of Fusarium oxysporum Fo47 compared with a previous assembly of Fo47 (GenBank accession GCA_000271705.2) and F. oxysporum f. sp. lycopersici Fol4287 (GenBank accession GCA_000149955.2)

\begin{tabular}{|c|c|c|c|}
\hline Strains & Fo47 (this study) & Fo47 (GCA_000271705.2) ${ }^{a}$ & Fol4287 ${ }^{b}$ \\
\hline Number of contigs & 12 & 124 & 88 \\
\hline Number of chromosomes & $12^{\mathrm{C}}$ & NA & 15 \\
\hline Number of lineage-specific chromosomes & 1 & NA & 4 \\
\hline Genome size $(\mathrm{Mb})$ & 50.36 & 49.67 & 61.47 \\
\hline GC content $(\%)$ & 47.71 & 47.68 & 48.48 \\
\hline Contig $\mathrm{N}_{50}(\mathrm{bp})$ & $4,523,040$ & $3,844,136$ & $4,589,937$ \\
\hline Number of protein-coding genes ${ }^{d}$ & 17,127 & 16,985 & 17,628 \\
\hline Repeat content $(\%)^{d}$ & 3.42 & 2.77 & 5.43 \\
\hline Predicted proteome completeness $(\%)^{\mathrm{e}}$ & 99 & 99 & 98 \\
\hline
\end{tabular}

a $\mathrm{NA}=$ unavailable.

b Reported by Ma et al. (2010).

c Number of putative centromeres was determined using Hi-C contact map.

d Protein-coding genes and repeat content were identified by the same pipeline (this study) to ensure a fair comparison.

e Completeness $=($ total BUSCOs - missing BUSCOs $) /$ total BUSCOs $\times 100$.

chromosomes) (Ma et al. 2010) was $46.10 \mathrm{Mb}$, suggesting a set of 11 core chromosomes in Fo47. A single Fo47 chromosome, Chr7 (4.23 Mb), did not align to any Fol4287 chromosome, indicative of a lineage-specific chromosome in Fo47. Chr7 is highly enriched for transposable elements (TEs), with $51 \%$ of total TE sequences located on Chr7 and $49 \%$ located on the rest of genome. Overrepresentation of repetitive sequences in this chromosome explains the fragmentation in the initial assembly. Chr7 has a smaller average gene density (290 per megabase) compared with core chromosomes (347 per megabase). The 1,229 genes on Chr7 are significantly enriched $(P$ value $=1.5 \mathrm{e}-4)$ for regulators of G-protein signaling functions, suggesting their likely role in external sensing and organism response to external nutrient sources and other environmental signals (Bayram and Braus 2012; Kim et al. 2017). This genome assembly of Fo47 will be a useful resource for comparative genomics of F. oxysporum and future functional genomic studies of Fo47 and host endophytic interactions. This genome project is indexed at GenBank under BioProject accession number PRJNA624237. The chromosomal sequence and gene annotation of Fo47 can be found at GenBank with accession numbers CP052038 to CP052049. PacBio reads, Illumina reads, and Hi-C Illumina data are available in the NCBI Sequenced Read Archive under the accession numbers SRR11526760, SRR11523115, and SRR11523120, respectively.

\section{Acknowledgments}

We thank the anonymous reviewers for their kind and helpful comments on the original manuscript.

\section{Author-Recommended Internet Resource}

NextDenovo: https://github.com/Nextomics/NextDenovo

\section{Literature Cited}

Aimé, S., Alabouvette, C., Steinberg, C., and Olivain, C. 2013. The endophytic strain Fusarium oxysporum Fo47: A good candidate for priming the defense responses in tomato roots. Mol. Plant-Microbe Interact. 26:918-926.

Alabouvette, C. 1986. Fusarium-wilt suppressive soils from the Châteaurenard region: Review of a 10-year study. Agronomie 6:273-284.

Alabouvette, C., Olivain, C., Migheli, Q., and Steinberg, C. 2009. Microbiological control of soil-borne phytopathogenic fungi with special emphasis on wiltinducing Fusarium oxysporum. New Phytol. 184:529-544.

Bayram, O., and Braus, G. H. 2012. Coordination of secondary metabolism and development in fungi: The velvet family of regulatory proteins. FEMS Microbiol. Rev. 36:1-24.
Belton, J. M., and Dekker, J. 2015. Hi-C in budding yeast. Cold Spring Harb. Protoc. 2015:649-661.

Borodovsky, M., and Lomsadze, A. 2011. Eukaryotic gene prediction using GeneMark.hmm-E and GeneMark-ES. Curr. Protoc. Bioinf. 4:4.6.1-4.6.10.

Cantarel, B. L., Korf, I., Robb, S. M., Parra, G., Ross, E., Moore, B., Holt, C., Sánchez Alvarado, A., and Yandell, M. 2008. MAKER: An easy-to-use annotation pipeline designed for emerging model organism genomes. Genome Res. 18:188-196.

de Lamo, F. J., and Takken, F. L. W. 2020. Biocontrol by Fusarium oxysporum using endophyte-mediated resistance. Front. Plant Sci. 11:37.

Durand, N. C., Robinson, J. T., Shamim, M. S., Machol, I., Mesirov, J. P., Lander, E. S., and Aiden, E. L. 2016a. Juicebox provides a visualization system for $\mathrm{Hi}-\mathrm{C}$ contact maps with unlimited zoom. Cell Syst. 3:99-101. 
Durand, N. C., Shamim, M. S., Machol, I., Rao, S. S., Huntley, M. H., Lander, E. S., and Aiden, E. L. 2016b. Juicer provides a one-click system for analyzing loopresolution Hi-C experiments. Cell Syst. 3:95-98.

Edel-Hermann, V., and Lecomte, C. 2019. Current status of Fusarium oxysporum formae speciales and races. Phytopathology 109:512-530.

$\mathrm{He}$, C. Y., Hsiang, T., and Wolyn, D. J. 2002. Induction of systemic disease resistance and pathogen defense responses in Asparagus officinalis inoculated with nonpathogenic strains of Fusarium oxysporum. Plant Pathol. 51:225-230.

Kim, Y., Heo, I. B., Yu, J. H., and Shin, K. S. 2017. Characteristics of a regulator of G-protein signaling (RGS) rgsC in Aspergillus fumigatus. Front. Microbiol. 8:2058.

Kistler, H. C. 1997. Genetic diversity in the plant-pathogenic fungus Fusarium oxysporum. Phytopathology 87:474-479.

Kurtz, S., Phillippy, A., Delcher, A. L., Smoot, M., Shumway, M., Antonescu, C., and Salzberg, S. L. 2004. Versatile and open software for comparing large genomes. Genome Biol. 5:R12.

Ma, L.-J., Geiser, D. M., Proctor, R. H., Rooney, A. P., O'Donnell, K., Trail, F., Gardiner, D. M., Manners, J. M., and Kazan, K. 2013. Fusarium pathogenomics. Annu. Rev. Microbiol. 67:399-416.

Ma, L.-J., van der Does, H. C., Borkovich, K. A., Coleman, J. J., Daboussi, M.-J., Di Pietro, A., Dufresne, M., Freitag, M., Grabherr, M., Henrissat, B., Houterman, P. M., Kang, S., Shim, W.-B., Woloshuk, C., Xie, X., Xu, J.-R., Antoniw, J., Baker, S. E., Bluhm, B. H., Breakspear, A., Brown, D. W., Butchko, R. A. E., Chapman, S., Coulson, R., Coutinho, P. M., Danchin, E. G. J., Diener, A., Gale, L. R., Gardiner, D. M., Goff, S., Hammond-Kosack, K. E., Hilburn, K., Hua-Van, A., Jonkers, W., Kazan, K., Kodira, C. D., Koehrsen, M., Kumar, L., Lee, Y.-H., Li, L., Manners, J. M., Miranda-Saavedra, D., Mukherjee, M., Park, G., Park, J., Park, S.-Y., Proctor, R. H., Regev, A., Ruiz-Roldan, M. C., Sain, D., Sakthikumar, S., Sykes, S., Schwartz, D. C., Turgeon, B. G., Wapinski, I., Yoder, O., Young, S., Zeng, Q., Zhou, S., Galagan, J., Cuomo, C. A., Kistler, H. C., and Rep, M. 2010. Comparative genomics reveals mobile pathogenicity chromosomes in Fusarium. Nature 464:367-373.

Marbouty, M., Cournac, A., Flot, J. F., Marie-Nelly, H., Mozziconacci, J., and Koszul, R. 2014. Metagenomic chromosome conformation capture (meta3C) unveils the diversity of chromosome organization in microorganisms. eLife 3:e03318.
Marçais, G., and Kingsford, C. 2011. A fast, lock-free approach for efficient parallel counting of occurrences of k-mers. Bioinformatics 27:764-770.

Michielse, C. B., and Rep, M. 2009. Pathogen profile update: Fusarium oxysporum. Mol. Plant Pathol. 10:311-324.

Paparu, P., Dubois, T., Coyne, D., and Viljoen, A. 2007. Defense-related gene expression in susceptible and tolerant bananas (Musa spp.) following inoculation with non-pathogenic Fusarium oxysporum endophytes and challenge with Radopholus similis. Physiol. Mol. Plant Pathol. 71: 149-157.

Pieterse, C. M., Zamioudis, C., Berendsen, R. L., Weller, D. M., Van Wees, S. C., and Bakker, P. A. 2014. Induced systemic resistance by beneficial microbes. Annu. Rev. Phytopathol. 52:347-375.

Simão, F. A., Waterhouse, R. M., loannidis, P., Kriventseva, E. V., and Zdobnov, E. M. 2015. BUSCO: Assessing genome assembly and annotation completeness with single-copy orthologs. Bioinformatics 31:3210-3212.

Stanke, M., and Morgenstern, B. 2005. AUGUSTUS: A web server for gene prediction in eukaryotes that allows user-defined constraints. Nucleic Acids Res. 33 (Web Server): W465-W467.

Tarailo-Graovac, M., and Chen, N. 2009. Using RepeatMasker to identify repetitive elements in genomic sequences. Curr. Protoc. Bioinf. 5:4.10.1-4.10.14.

Van der Ent, S., Van Wees, S. C. M., and Pieterse, C. M. J. 2009. Jasmonate signaling in plant interactions with resistance-inducing beneficial microbes. Phytochemistry 70:1581-1588.

Veloso, J., and Díaz, J. 2012. Fusarium oxysporum Fo47 confers protection to pepper plants against Verticillium dahliae and Phytophthora capsici and induces the expression of defense genes. Plant Pathol. 61:281-288.

Vurture, G. W., Sedlazeck, F. J., Nattestad, M., Underwood, C. J., Fang, H., Gurtowski, J., and Schatz, M. C. 2017. GenomeScope: Fast reference-free genome profiling from short reads. Bioinformatics 33:2202-2204.

Walker, B. J., Abeel, T., Shea, T., Priest, M., Abouelliel, A., Sakthikumar, S., Cuomo, C. A., Zeng, Q., Wortman, J., Young, S. K., and Earl, A. M. 2014. Pilon: An integrated tool for comprehensive microbial variant detection and genome assembly improvement. PLoS One 9:e112963. 